OPEN ACCESS

Edited by:

Zsolt Illes,

University of Southern Denmark

Odense, Denmark

Reviewed by:

Gabor Lovas,

Jahn Ferenc Dél-Pesti Kórház és

Rendelőintézet, Hungary

Zsolt Mezei,

Aalborg University, Denmark

${ }^{*}$ Correspondence:

Yan Xu

xuyanpumch@hotmail.com

Specialty section:

This article was submitted to Multiple Sclerosis and Neuroimmunology,

a section of the journal

Frontiers in Neurology

Received: 15 July 2017 Accepted: 18 October 2017 Published: 03 November 2017

Citation:

Zhang Y, Fan S, Han F and Xu Y (2017) Paroxysmal Symptoms As the First Manifestation of

Multiple Sclerosis Mimicking a

Transient Ischemic Attack:

A Report of Two Cases.

Front. Neurol. 8:585.

doi: 10.3389/fneur.2017.00585

\section{Paroxysmal Symptoms As the First Manifestation of Multiple Sclerosis Mimicking a Transient Ischemic Attack: A Report of Two Cases}

\author{
Yao Zhang ${ }^{1}$, Siyuan Fan', Fei Han' ${ }^{2}$ and Yan Xu ${ }^{1 *}$ \\ ${ }^{1}$ Multiple Sclerosis Center, Department of Neurology, Peking Union Medical College Hospital, Peking Union Medical College, \\ Chinese Academy of Medical Sciences, Beijing, China, ${ }^{2}$ Department of Neurology, Peking Union Medical College Hospital, \\ Peking Union Medical College, Chinese Academy of Medical Sciences, Beijing, China
}

Paroxysmal symptoms are unusual manifestations of multiple sclerosis (MS). When presented as the first clinical manifestation, paroxysmal symptoms may easily be mistaken for transient ischemic attack (TIA). Previously, several cases of MS that reported with paroxysmal symptoms were misdiagnosed as TIA. Here, we report two additional cases, focusing on the clinical characteristics of paroxysmal symptoms in MS. Both cases had paroxysmal symptoms as their first manifestation; one presented with transient dizziness, left face numbness, and right limb weakness, and the other presented with episodic lightheadedness, blurred vision, nausea, palpitations, and tremulousness upon standing. Both of the patients' symptoms were mistaken for TIA at first, based on microembolic signals recorded by transcranial Doppler, but were later correctly diagnosed with MS based on neuroimaging and lumbar puncture. The paroxysmal symptoms responded to carbamazepine and were relieved completely after administration of intravenous methylprednisolone and interferon. Herein, we aim to summarize the differences between paroxysmal symptoms seen in MS and TIA, to facilitate a timely differential diagnosis and recommend an early appropriate treatment.

Keywords: paroxysmal symptoms, multiple sclerosis, transient ischemic attack, transcranial Doppler, mimic

\section{BACKGROUND}

Multiple sclerosis (MS) is the most common immune-mediated inflammatory demyelinating disease of the central nervous system. The characteristic neuropathological feature of MS is the presence of focal demyelinated plaques within white matter of the central nervous system. Based on this pathological feature, the classic clinical manifestations of MS include optic neuritis, long tract symptoms (e.g., numbness or weakness), rather than transient symptoms as in epilepsy and transient ischemic attack (TIA). However, paroxysmal symptoms have occasionally been reported in MS since in 1959 (1). These transient symptoms are characterized by their brevity, frequency, stereotyped fashion, and response to carbamazepine (2). If untreated they tend to continue in clusters for days up to a few months. Paroxysmal symptoms in MS, especially when presented as the first clinical manifestation, could be mistaken for TIA, epilepsy, and neuralgias (3). Thus, identifying paroxysmal symptoms as a possible first clinical symptom of MS is important for differential diagnosis from TIA, as well as for early diagnosis and treatment of the disease. We report two cases of MS with paroxysmal 
symptoms as their first manifestation and review the literature. Both patients were misdiagnosed with TIA before receiving the proper diagnosis of MS.

\section{CASE PRESENTATION 1}

A 32-year-old male developed episodic, transient, sudden-onset dizziness, left face numbness, and right limb weakness. He experienced the attacks 20-30 times a day, with each episode lasting $10-20 \mathrm{~s}$. He was completely well between the attacks. The patient was admitted to our emergency department with the suspicion of TIA. Cardiovascular risk factors included cigarette smoking of 5-6 cigarettes/day for 10 years. There was no history of migraines, and his neurological exam was normal at admission.

\section{DESCRIPTION OF LABORATORY INVESTIGATIONS AND DIAGNOSTIC TESTS}

A head CT excluded intracranial hemorrhage and subarachnoid hemorrhage. With the suspicion of TIA, extracranial, intracranial arteries, and the heart were studied. The cardiovascular examinations, including extra- and intracranial ultrasound and transthoracic echocardiography, were normal. Transcranial Doppler (TCD) contrast agent was used to detect intracardiac shunts, and three microembolic signals were recorded on TCD spectrum by $2 \mathrm{~Hz}$ probes within $25 \mathrm{~s}$ of contrast injection. The preliminary diagnosis was cryptogenic stroke with patent foramen ovale (PFO), and the patient was treated with aspirin $(100 \mathrm{mg} /$ day $)$ for 7 days, but his episodes persisted. To exclude ischemic stroke and other related disease, brain magnetic resonance imaging (MRI), including six sequences (T1-weighted axial, T2-weighted sagittal, T2-weighted axial, FLAIR, DWI, and ADC) as well as T1-weighted contrast-enhanced images was done 1 week later. The MRI showed an asymptomatic gadolinium-enhancing lesion in the juxtacortical region of the frontal lobe on T1-weighted images with gadolinium enhancement. Several hyperintense lesions in the midbrain, pons, and periventricular region had also been found on T2-weighted images without gadolinium enhancement (Figure 1). The serum autoantibody test was negative, and connective tissue diseases were ruled out. During this episodes, his electroencephalogram was normal, and epileptic seizure was ruled out. The patient was suspected of demyelinating diseases, and lumbar punctures was done. Cerebrospinal fluid (CSF) cell counts and protein levels were normal, but isoelectric focusing revealed oligoclonal IgG bands (OCBs). All of these results were consistent with the McDonald criteria, and the attacks were qualified as paroxysmal symptoms of MS. The patient was treated with intravenous methylprednisolone (1,000 mg) for 5 days as well as carbamazepine $(200 \mathrm{mg} /$ day $)$. His attacks promptly decreased to less than 10 times per day. He was subsequently started on interferon, and his attacks resolved completely 3 weeks later. The carbamazepine was discontinued after 3 months, and he was relapse-free during his 1-year follow-up. Unfortunately, he did not do follow-up MRI in our hospital after interferon was administered.

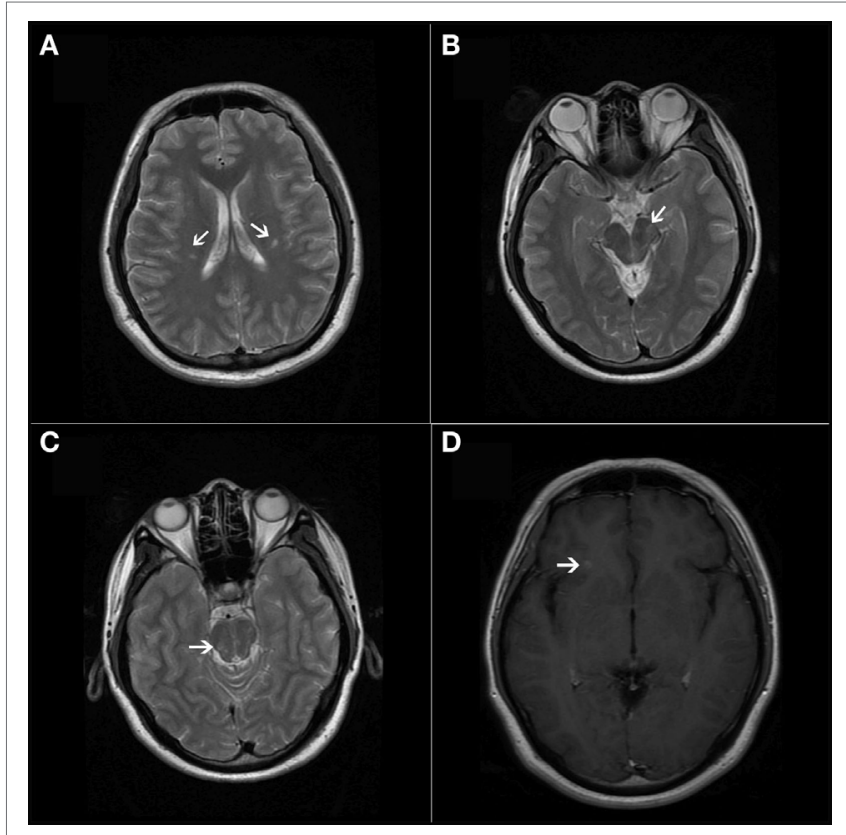

FIGURE 1 | (A-C) T2-weighted axial magnetic resonance imaging showed lesions in bilateral periventricular and infratentorial areas of the CNS.

(D) T1-weighted contrast-enhanced image showed a simultaneous presence of asymptomatic gadolinium-enhancing lesion at the juxtacortical area of the anterior part of the insular cortex.

\section{CASE PRESENTATION 2}

A 16-year-old female with no cardiovascular risk factors presented with episodic and sudden-onset lightheadedness, blurred vision, nausea, palpitations, and tremulousness upon standing. Syncope occurred several times when she stood up quickly. She experienced such episodes 7-10 times daily, each lasting less than $1 \mathrm{~min}$. Her past medical history was clear, and her neurological exam was normal at admission.

\section{DESCRIPTION OF LABORATORY INVESTIGATIONS AND DIAGNOSTIC TESTS}

Since she was suspected of TIA at admission, MRI, carotid ultrasound, and TCD were done the next day. The T2-weighted MRI of her brain revealed multiple, ovoid-shaped, hyperintense lesions in the periventricular region, the inferior temporal lobe white matter, and the pons. No gadolinium-enhancing lesion was found on T1-weighted contrast-enhanced image. The carotid ultrasound was normal but 17 microembolic signals were recorded by $4 \mathrm{~Hz}$ probes on TCD spectrum within $6 \mathrm{~s}$, while other cardiovascular examinations were normal. She was suspected of TIA, PFO, and was treated with aspirin $(100 \mathrm{mg} /$ day $)$. However, she later experienced even more episodes, approximately 20-30 times per day. The episodes resolved gradually 2 months later, but after 6 months had passed, the episodes returned. A follow-up MRI revealed new T2-hyperintense lesions in characteristic locations (Figure 2): periventricular, juxtacortical, and infratentorial regions, as well 


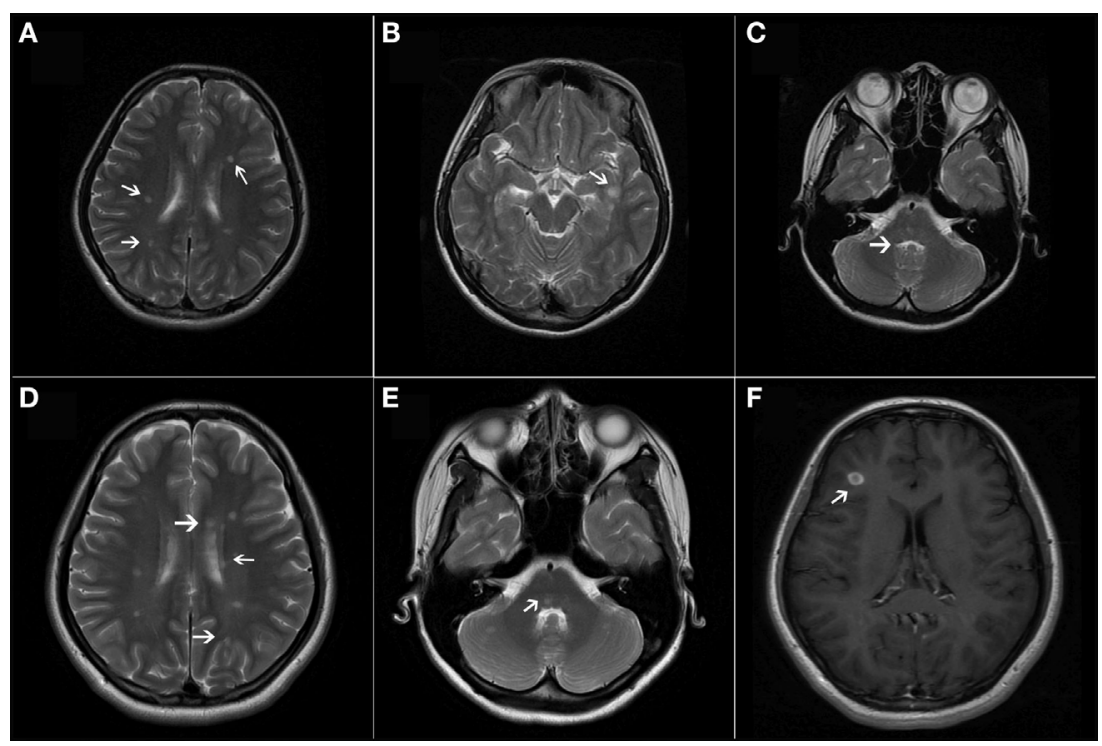

FIGURE 2 | (A-C) T2-weighted axial magnetic resonance imaging (MRI) revealed multiple, ovoid-shaped, hyperintense lesions in the bilateral periventricular regions, the juxtacortical area of the left inferior temporal lobe, and the pons. (D,E) T2-weighted axial MRI showed new lesions in the periventricular regions and the cerebellum. (F) T1-weighted contrast-enhanced image showed one asymptomatic gadolinium-enhancing lesion in the right frontal lobe.

as one gadolinium-enhancing lesion on the frontal lobe. MS was suspected, and lumbar puncture was done. The CSF total leukocyte count and protein level were normal but isoelectric focusing revealed OCBs different from bands in serum. In a head-up tilt table test, she demonstrated a sustained heart rate increase of 52 beats per minute (bpm) within the first $10 \mathrm{~min}$ of tilt (from 68 to $120 \mathrm{bpm}$ ) without orthostatic hypotension. She was diagnosed with MS and postural tachycardia syndrome. The lesion on the pons was responsible for her vegetative instability. Intravenous methylprednisolone (1,000 mg) was administered for 5 days, and carbamazepine (200 mg/day) was also started. Her paroxysmal symptoms resolved promptly, and 3 weeks later carbamazepine was stopped. She was subsequently started on interferon beta- $1 \mathrm{~b}$ ( $250 \mu \mathrm{g}$ every other day). She reported that the same episodes recurred when she discontinued interferon half a year later. Since there were no other neurological symptoms or increased disability, steroid treatment was not initiated. Her episodes resolved again after continuation of interferon.

\section{DISCUSSION}

Paroxysmal symptoms are unusual manifestations of MS, especially at onset of the disease. An electronic case database was established at the MS clinic of the Department of Neurology, Peking Union Medical College Hospital in 2011 (4). By December 2016 , a total of 520 MS patients had been included, yet only 4 $(0.77 \%)$ cases presented with paroxysmal syndromes as their first clinical manifestation. This rate is comparable to the previous largest study that reported $1.1 \%(15 / 1,396)$ of MS patients presented with paroxysmal symptoms (5). A population-based, MS cohort study reported the yearly incidence of brainstem and spinal paroxysmal symptoms was 190 cases in 10,000 MS patients (6).
When paroxysmal symptoms are the presenting manifestation of MS, it can be challenging for clinicians to differentiate these symptoms from TIA. Several cases of paroxysmal symptoms in MS have been reportedly mistaken for TIA $(7,8)$. We found in agreement with these reports that paroxysmal symptoms in MS had several typical characteristics that can be differentiated from TIA (Table 1). Paroxysmal symptoms in MS are characterized by their sudden onset, brevity (usually seconds to minutes) (5), frequency (from 10 to 20 times per day up to a few hundred times per day), stereotyped fashion, and relatively long clinical course. If untreated, they tend to continue in clusters for days up to a few months before remission (7), coincident with a new episode of inflammatory demyelination. Relapses in MS have been defined as episodes of neurological disturbances last for at least $24 \mathrm{~h}$. As a consequence, paroxysmal symptoms are accepted as relapses as long as they consist of multiple episodes occurring over not less than $24 \mathrm{~h}$. These transient symptoms usually have a positive response to carbamazepine, while TIA does not.

Patent foramen ovale, which can be detected in about $25 \%$ of the healthy population, is a common anatomical variant leading to right-to-left shunt (RLS) (9). Compared to transesophageal echocardiography (TEE), the gold standard of RLS detection, TCD is a faster, less expensive, non-invasive bedside method for the detection of RLS in patients with cryptogenic cerebral ischemia and TIA. The sensitivity and specificity of TCD in the detection of PFO have been reported to be 96.1 and $92.4 \%$, respectively. Compared to TCD, TEE is superior in terms of higher positive likelihood ratio values, which suggests that TCD is less specific for the detection of PFO (10). TCD diagnoses right-to-left atrial shunt (RLS) by detection of microbubbles $(\mathrm{MB})$ passing through the middle cerebral artery. The threshold value of $42 \mathrm{MB}$ during normal breathing was suggested to discriminate shunts related to stroke or TIA from a "bystander PFO" with no pathophysiological relationship to the 
TABLE 1 | Comparison of clinical manifestations between paroxysmal symptoms and transient ischemic attack (TIA).

\begin{tabular}{lll}
\hline & Paroxysmal symptoms & TIA \\
\hline Trigger & $\begin{array}{l}\text { Can be triggered by sensory stimuli, } \\
\text { hyperventilation, or movement (7) }\end{array}$ & $\begin{array}{l}\text { Decreased perfusion } \\
\text { pressure }\end{array}$ \\
\hline Brevity & Lasting seconds to minutes & Lasting minutes to hours \\
\hline Frequency & $\begin{array}{l}\text { From 10 to 20 times per day up to a } \\
\text { few hundred times per day }\end{array}$ & $\begin{array}{l}\text { Less than } 10 \text { times per } \\
\text { day, even once per week }\end{array}$ \\
\hline Clinical course & $\begin{array}{l}\text { Tend to continue in clusters with } \\
\text { great intensity for days up to a few } \\
\text { months. Followed by remission }\end{array}$ & $\begin{array}{l}\text { Continue for days and } \\
\text { sometimes proceed to } \\
\text { stroke }\end{array}$ \\
\hline Response to & Good response & No response \\
carbamazepine & &
\end{tabular}

symptoms (11). Both of the two cases in this report had considerably less than $42 \mathrm{MB}$ ( $3 \mathrm{MB}$ in case 1 , and $17 \mathrm{MB}$ in case 2 ) detected by TCD, and the RLS had no clinical significance.

Several clinical types of paroxysmal symptoms have been described (3), including paroxysmal dysarthria with ataxia, paroxysmal diplopia, paroxysmal paresthesia, tonic spasm, and paroxysmal hemiparesis. Different clinical manifestations reflect the disruption of axonal conduction in different areas of the central nervous system. The mechanisms of paroxysmal symptoms in MS are not fully understood, but are likely mediated by ephaptic transmission (12). On this basis, membrane-stabilizing drugs are effective in almost all patients (7). The paroxysmal symptoms have a tendency to occur in the early stages of the MS disease course (2). A possible explanation for this is that only the intact naked axons that exist in the early stages of MS and that feature relatively less inflammatory damage could create paroxysmal symptoms, by spreading the neuronal conduction transversely and activating adjacent anatomical structures (2).

\section{CONCLUSION}

Paroxysmal symptoms that present as the first manifestation of MS may easily be diagnosed as TIA. Paroxysmal symptoms

\section{REFERENCES}

1. Andermann F, Cosgrove JB, Lloyd-Smith D, Walters AM. Paroxysmal dysarthria and ataxia in multiple sclerosis; a report of 2 unusual cases. Neurology (1959) 9(4):211-5. doi:10.1212/WNL.9.4.211

2. Tuzun E, Akman-Demir G, Eraksoy M. Paroxysmal attacks in multiple sclerosis. Mult Scler (2001) 7(6):402-4. doi:10.1177/135245850100700609

3. Matthews WB. Paroxysmal symptoms in multiple sclerosis. J Neurol Neurosurg Psychiatry (1975) 38(6):617-23. doi:10.1136/jnnp.38.6.617

4. Xu Y, Wang Q, Ren HT, Qiao L, Zhang Y, Fei YY, et al. Comparison of efficacy and tolerability of azathioprine, mycophenolate mofetil, and cyclophosphamide among patients with neuromyelitis optica spectrum disorder: a prospective cohort study. J Neurol Sci (2016) 370:224-8. doi:10.1016/j.jns. 2016.09.035

5. Ehling R, Bsteh G, Di Pauli F, Hegen H, Auer M, Obermair K, et al. Rethinking the importance of paroxysmal and unusual symptoms as first clinical manifestation of multiple sclerosis: they do matter. Mult Scler Relat Disord (2016) 9:150-4. doi:10.1016/j.msard.2016.07.014 in MS can be differentiated from TIA by their brevity (usually seconds to minutes), frequency (10-20 times per day up to a few hundred times per day), relatively long-clinical course (tend to continue in clusters for days up to a few months), and positive response to carbamazepine. Differential diagnosis of MS cases presented with paroxysmal symptoms from TIA is important for early diagnosis of MS and early initiation of the diseasemodifying therapies.

\section{ETHICS STATEMENT}

No interventions were performed outside routine clinical care for these two patients. No formal research ethics approval was required because this is a case report and there was no experimental intervention into routine care. This study was carried out in accordance with the recommendations of Ethics Committee of Peking Union Medical College Hospital with written informed consent from all subjects. All subjects gave written informed consent to participate in the study in accordance with the Declaration of Helsinki. The protocol was approved by the Ethics Committee of Peking Union Medical College Hospital. Written informed consent was obtained from all the participants for the publication of this case report.

\section{AUTHOR CONTRIBUTIONS}

SF and FH conducted the patients' investigations and provided clinical care, reviewed the manuscript, and approved the final manuscript as submitted. YZ planned the case report, drafted the initial manuscript, reviewed and revised the manuscript, and approved the final manuscript as submitted. YX helped plan clinical investigations, critically reviewed and revised the manuscript, and approved the final manuscript as submitted.

\section{ACKNOWLEDGMENTS}

We are grateful to the two patients and their families who participated in this case report.

6. Eriksson M, Ben-Menachem E, Andersen O. Epileptic seizures, crania neuralgias and paroxysmal symptoms in remitting and progressive multiple sclerosis. Mult Scler (2002) 8(6):495-9. doi:10.1191/1352458502ms812oa

7. Yates TJ, Crawley F. Paroxysmal symptoms in multiple sclerosis masquerading as transient ischaemicattacks. BMJ Case Rep (2010)2010.doi:10.1136/bcr.03.2010.2831

8. Perren F, Gschwind M, Landis T, Kremer C, Lalive P. Multiple sclerosis relapse mimicking a transient ischemic attack. Mult Scler Relat Disord (2016) 5:27-8. doi:10.1016/j.msard.2015.10.003

9. Hagen PT, Scholz DG, Edwards WD. Incidence and size of patent foramen ovale during the first 10 decades of life: an autopsy study of 965 normal hearts. Mayo Clin Proc (1984) 59(1):17-20. doi:10.1016/S0025-6196(12)60336-X

10. Katsanos AH, Psaltopoulou T, Sergentanis TN, Frogoudaki A, Vrettou AR, Ikonomidis I, et al. Transcranial Doppler versus transthoracic echocardiography for the detection of patent foramen ovale in patients with cryptogenic cerebral ischemia: a systematic review and diagnostic test accuracy meta-analysis. Ann Neurol (2016) 79(4):625-35. doi:10.1002/ana.24609

11. Tanzi A, Onorato E, Casilli F, Anzola GP. Is the search for right-to-left shunt still worthwhile? Acta Neurol Scand (2016) 133(4):281-8. doi:10.1111/ane.12456 
12. Baker $\mathrm{M}$, Bostock $\mathrm{H}$. Ectopic activity in demyelinated spinal root axons of the rat. J Physiol (1992) 451:539-52. doi:10.1113/jphysiol.1992.sp019178

Conflict of Interest Statement: The authors declare that the research was conducted in the absence of any commercial or financial relationships that could be construed as a potential conflict of interest.
Copyright $\odot 2017$ Zhang, Fan, Han and Xu. This is an open-access article distributed under the terms of the Creative Commons Attribution License (CC BY). The use, distribution or reproduction in other forums is permitted, provided the original author(s) or licensor are credited and that the original publication in this journal is cited, in accordance with accepted academic practice. No use, distribution or reproduction is permitted which does not comply with these terms. 